dass das Risiko über die Behandlungszeit ansteigt [2], sodass es hier zu einer weiteren Datenverzerrung kommt. Eine weitere Beschränkung liegt, wie von den Autoren selbst diskutiert, in der Anwendung von QALYs, die abstrakt sind und eine Translation in den Alltag schwierig machen.

Zusammenfassend handelt es sich um einen interessanten Ansatz, eine Stratifizierung von Therapieoptionen vorzunehmen und eine Rationale für die Einleitung einer MS-Therapie zu haben, der es ermöglicht mehrere Therapien gegeneinander zu testen. Da jedoch die wenigsten Patienten der "Modellpatientin" ähneln und Verläufe sehr individuell sind, bleibt eine Risikomodellierung zu abstrakt, um hierauf individuelle Therapieentscheidungen zu gründen.
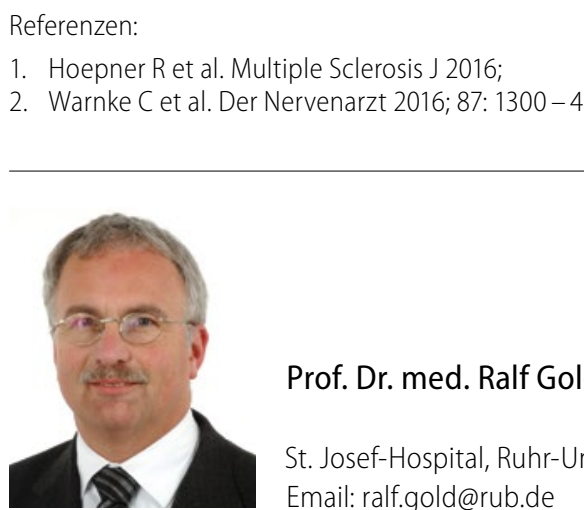

Prof. Dr. med. Ralf Gold, Bochum

St. Josef-Hospital, Ruhr-Universität Bochum Email: ralf.gold@rub.de

\title{
Erfolg der Thrombektomie unabhängig von Sedierung oder Narkose
}

Fragestellung: Beeinflusst das periprozedurale Management den Erfolg der Thrombektomie beim ischämischen Insult?

Hintergrund: Die Thrombektomie ist nach sechs positiven Studien jetzt die Standardtherapie bei schweren Schlaganfällen mit Verschlüssen der distalen A. carotis interna oder der proximalen A. cerebri media. Die Thrombektomie erfolgt in aller Regel zusätzlich zur systemischen Thrombolyse. Post-hoc-Analysen der bisher durchgeführten Studien legten nahe, dass möglicherweise die Thrombektomie unter Sedierung einen besseren Outcome hat als die Thrombektomie unter Intubationsnarkose. Die Studie SIESTA (Sedations vs. Intubation for Endovascular Stroke Treatment) sollte diese Frage in einem randomisierten Design untersuchen.

Schönenberger S, Uhlmann L, Hacke W et al. Effect of conscious sedation vs general anesthesia on early neurological improvement among patients with ischemic stroke undergoing endovascular thrombectomy: A randomized clinical trial. JAMA 2016; 316: $1986-96$
Patienten und Methodik: Die Studie wurde als randomisierte Parallelgruppenstudie an der Neurologischen Universitätsklinik in Heidelberg durchgeführt. Eingeschlossen wurden 150 Patienten mit ischämischen In- sulten in der vorderen Zirkulation und einem NIHSS-Score von über 10. In der Gruppe mit Sedierung erhielten die Patienten Analgetika und Sedativa, in der Parallelgruppe wurden die $\mathrm{Pa}$ tienten intubiert und erhielten eine Vollnarkose. Der primäre Endpunkt der Studie war die Verbesserung der neurologischen Ausfälle auf der NIHSS-Skala nach 24 Stunden. Sekundäre Endpunkte waren der funktionelle Outcome gemessen mit der modifizierten Rankin-Skala nach drei Monaten sowie Sterblichkeit und Sicherheit.

Ergebnisse: 150 Patienten mittleren Alters von 71,5 Jahren wurden in die Studie eingeschlossen, davon 60 Frauen. Die Schlaganfallschwere lag bei einem medianen NIHSS-Score von 17. Die Verbesserung des NIHSS-Scores nach 24 Stunden war mit 3,2 Punkten in der Narkose- versus 3,6 Punkten in der Sedierungsgruppe nicht unterschiedlich. Auch die sekundären Endpunkte waren nicht unterschiedlich. In der Narkosegruppe kam es signifikant häufiger zu einer Hypothermie und Pneumonie.

Schlussfolgerungen: Patienten mit akutem ischämischem Insult, die eine Thrombektomie erhalten, haben denselben funktionellen Outcome unabhängig davon, ob der Eingriff in Allgemeinnarkose oder in Sedierung erfolgt.

\section{- Kommentar von Hans-Christoph Diener, Essen}

\section{Klinisch sehr wichtige Ergebnisse}

Die gut durchgeführte randomisierte Studie aus Heidelberg zeigt erneut, wie wichtig es ist, klinische Fragestellungen durch randomisierte Studien zu untersuchen und sich nicht auf die Post-hoc-Analysen von Studien mit anderen Fragestellungen zu verlassen. Post-hoc-Analysen können nie ausschließen, dass

schwer betroffene Patienten oder Patienten, die motorisch unruhig sind, eher eine Vollnarkose erhalten als eine Sedierung. Die Ergebnisse aus Heidelberg sind sehr wichtig, da die Notwendigkeit einer Vollnarkose bei sehr unruhigen Patienten keinen therapeutischen Nachteil nach sich zieht. 